PANORAMA

ISSN: 1909-7433

ISSN: 2145-308X

ednorman@poligran.edu.co

Politécnico Grancolombiano

Colombia

\title{
INNOVACIONES EDUCATIVAS: PERSPECTIVAS DE DOCENTES Y ESTUDIANTES DE LA UNIVERSIDAD NACIONAL DE RÍO CUARTO (ARGENTINA) Y LA UNIVERSIDAD DEL ATLANTICO (COLOMBIA)
}

Moreira, Clarisa; Abuzaid, Jael Naim; Elisondo, Romina Cecilia; Melgar, María Fernanda

INNOVACIONES EDUCATIVAS: PERSPECTIVAS DE DOCENTES Y ESTUDIANTES DE LA UNIVERSIDAD

NACIONAL DE RÍO CUARTO (ARGENTINA) Y LA UNIVERSIDAD DEL ATLÁNTICO (COLOMBIA)

PANORAMA, vol. 14, núm. 26, 2020

Politécnico Grancolombiano, Colombia

Disponible en: http://www.redalyc.org/articulo.oa?id=343963784003

DOI: https://doi.org/10.15765/pnrm.v14i26.1480

Esta obra está bajo una Licencia Creative Commons Atribución-NoComercial-SinDerivar 4.0 Internacional. 
PANORAMA, vol. 14, núm. 26, 2020

Politécnico Grancolombiano, Colombia

Recepción: 30 Noviembre 2019 Aprobación: 10 Febrero 2020

DOI: https://doi.org/10.15765/ pnrm.v14i26.1480

Redalyc: http://www.redalyc.org/ articulo.oa?id=343963784003
Artículos de investigación científica y tecnológica

INNOVACIONES EDUCATIVAS: PERSPECTIVAS DE DOCENTES Y ESTUDIANTES DE LA UNIVERSIDAD NACIONAL DE RÍO CUARTO (ARGENTINA) Y LA UNIVERSIDAD DEL ATLÁNTICO (COLOMBIA)

\author{
EDUCATIONAL INNOVATIONS: PERSPECTIVES OF \\ TEACHERS AND STUDENTS AT THE UNIVERSIDAD \\ NACIONAL DE RÍO CUARTO (ARGENTINA) AND \\ THE UNIVERSIDAD DEL ATLÁNTICO (COLOMBIA) \\ INOVAÇÕES EDUCATIVAS: PERSPECTIVAS DOS \\ PROFESSORES E ESTUDANTES DA UNIVERSIDADE \\ NACIONAL DE RIO CUARTO (ARGENTINA) E DA \\ UNIVERSIDAD DEL ATLÁNTICO (COLÔMBIA) \\ Clarisa Moreira clarisamoreira94@gmail.com \\ Universidad Nacional de Río Cuarto, Argentina \\ Jael Naim Abuzaid jaelabuzaid@gmail.com \\ Universidad Nacional de Río Cuarto, Argentina \\ Romina Cecilia Elisondo relisondo@gmail.com \\ Universidad Nacional de Río Cuarto, Argentina \\ María Fernanda Melgar fernandamelgar@gmail.com \\ Universidad Nacional de Río Cuarto, Argentina
}

Resumen: Innovar en las universidades es indispensable, considerando los constantes cambios y la complejidad de las problemáticas sociales actuales. Compete al presente estudio analizar, desde la perspectiva de docentes y estudiantes, condiciones que potencian y obstaculizan las innovaciones educativas en la universidad. En el marco de estas condiciones, se indaga respecto del papel de las tecnológicas como herramientas para la innovación. Se desarrolla un estudio cualitativo donde se recuperan los significados construidos por docentes y estudiantes de dos universidades latinoamericanas: la Universidad Nacional de Río Cuarto (UNRC), de Argentina, y la Universidad del Atlántico (UA), de Colombia. Participaron del estudio 18 docentes (11 de la UA y 7 de la UNRC), y 32 estudiantes (22 de la UA y 10 de la UNRC), de las carreras de Licenciatura en Educación especial y Licenciatura en Psicopedagogía. Como instrumentos de recolección de datos se utilizaron entrevistas y cuestionarios abiertos. Los análisis indican los siguientes aspectos facilitadores de la innovación: formación docente continua, investigación de las innovaciones, políticas institucionales, trabajo en equipo, incorporación de tecnologías y actividades más allá de las aulas. Resistencias al cambio, burocratización, multiplicidad de tareas, masividad y falta de tiempo aparecen como aspectos obstaculizadores de las innovaciones, según los docentes y los estudiantes que participaron del estudio. El propósito es generar conocimientos que contribuyan 
a la comprensión de las innovaciones educativas como campo complejo. También se espera realizar aportes para el diseño y la ejecución de propuestas y políticas educativas orientadas a la transformación de las universidades.

Palabras clave: Universidad, innovación educativa, tecnologías, aprendizaje, enseñanza. Resumo: Inovar nas universidades é indispensável, tendo em conta as constantes mudanças e a complexidade dos problemas sociais actuais. Estamos interessados neste estudo para analisar, na perspectiva de professores e alunos, as condições que melhoram e dificultam as inovações educativas na universidade. No âmbito destas condições, investigamos o papel das tecnologias como instrumentos de inovação. Desenvolvemos um estudo qualitativo onde recuperamos os significados construídos por professores e estudantes de duas universidades latino-americanas: a Universidade Nacional do Rio Cuarto (UNRC) da Argentina e a Universidade do Atlântico (UA) da Colômbia. Participaram no estudo 18 professores ( 11 da UA e 7 da UNRC) e 32 estudantes (22 da UA e 10 da UNRC) da Licenciatura em Educação Especial e da Licenciatura em Psicopedagogia. Como instrumentos de recolha de dados, utilizámos entrevistas e questionários abertos. As análises indicam os seguintes aspectos facilitadores da inovação: formação contínua de professores, investigação em inovação, políticas institucionais, trabalho de equipa, incorporação de tecnologias e actividades fora da sala de aula. A resistência à mudança, a burocratização, a multiplicidade de tarefas, a massividade e a falta de tempo parecem estar a dificultar aspectos da inovação, segundo os professores e alunos que participaram no estudo. O nosso objectivo é gerar conhecimento que contribua para a compreensão das inovações educativas como um campo complexo. Esperamos poder contribuir para a concepção e implementação de propostas e políticas educativas que visem a transformação das universidades.

Palavras-chave: Universidade, inovação educativa, tecnologias, aprendizagem, ensino.

Abstract: Innovating in universities is indispensable considering the constant changes and the complexity of current social problems. We are interested in this study to analyze, from the perspective of teachers and students, conditions that enhance and hinder educational innovations at the university. Within the framework of these conditions, we investigate the role of technologies as tools for innovation. We developed a qualitative study where we recovered the meanings built by teachers and students of two Latin American universities: The National University of Rio Cuarto (UNRC) of Argentina and the University of the Atlantic (UA) of Colombia. Eighteen professors (11 from the UA and 7 from the UNRC) and 32 students ( 22 from the UA and 10 from the UNRC) from the bachelor's degree in Special Education and the Bachelor's degree in Psychopedagogy participated in the study. As data collection instruments we used interviews and open questionnaires. The analyses indicate the following facilitating aspects of innovation: continuous teacher training, innovation research, institutional policies, teamwork, incorporation of technologies and activities beyond the classroom. Resistance to change, bureaucratization, multiplicity of tasks, massiveness, and lack of time appear to be hampering aspects of innovation according to teachers and students who participated in the study. Our purpose is to generate knowledge that contributes to the understanding of educational innovations as a complex field. We hope to make contributions to the design and implementation of educational proposals and policies aimed at transforming universities.

Keywords: University, educational innovation, technologies, learning, teaching.

\section{Introducción}

Los acelerados cambios sociales, las múltiples y complejas formas actuales de comunicación y construcción de conocimientos demandan a las instituciones educativas innovaciones en las prácticas, en los planes de estudio y en las formas de comprender los procesos de enseñanza y aprendizaje. Las escuelas y las universidades, en tanto instituciones sociales, se configuran y reconfiguran en esta compleja y dinámica trama 
actual, donde las mediaciones tecnológicas juegan un papel relevante en la vida de las personas. En el campo educativo, las tecnologías pueden constituirse en herramientas para la democratización de los conocimientos y la construcción de instituciones educativas y sociedades más inclusivas (Norman-Acevedo, 2019).

Según la UNESCO, la innovación educativa es:

(...) un acto deliberado y planificado de solución de problemas, que apunta a lograr mayor calidad en los aprendizajes de los estudiantes, superando el paradigma tradicional. Implica trascender el conocimiento academicista y pasar del aprendizaje pasivo del estudiante a una concepción donde el aprendizaje es interacción y se construye entre todos. $(2016$, p. 3)

Es pertinente citar la definición de la UNESCO, porque permite sintetizar algunos aspectos importantes de la innovación educativa: cambio, resolución de problemas, papel activo de los estudiantes e importancia de las interacciones sociales en la enseñanza y el aprendizaje. Igualmente, es relevante destacar el papel que le asigna el organismo internacional a las innovaciones educativas como camino propicio para la transformación de los contextos de enseñanza y aprendizaje.

Interesa para el presente estudio analizar, desde la perspectiva de docentes y estudiantes, condiciones que potencian y obstaculizan las innovaciones educativas en la universidad. En el marco de estas condiciones, se indagó el papel de las tecnológicas como herramientas para la innovación. Se desarrolló un estudio cualitativo en el que se recuperó los significados construidos por docentes y estudiantes de dos universidades latinoamericanas: Universidad Nacional de Río Cuarto (Argentina), y la Universidad del Atlántico (Colombia). El estudio no pretende realizar generalizaciones, sino analizar perspectivas de los actores y construir conocimientos sobre los casos investigados. El propósito es generar conocimientos que contribuyan a la comprensión de las innovaciones educativas en la universidad como un campo complejo y dinámico. También se espera realizar contribuciones prácticas orientadas al diseño y la ejecución de propuestas educativas innovadoras.

\section{Marco teórico}

Las innovaciones son percibidas como rupturas o cambios en los supuestos y prácticas de actores e instituciones, que no son al azar o de forma deliberada, sino que suponen una intención y una planificación, y que están orientadas a mejorar situaciones que en un inicio se deducen como problemáticas (Macchiarola, 2009). Siguiendo esta línea, Rivas et al. (2017), agregan que este tipo de acciones planificadas movilizan cambios profundos basados en el deseo y la pasión por enseñar y aprender; consideran, además, que las innovaciones educativas implican rupturas o cambios en lo referido a características de la educación tradicional, como por ejemplo la composición memorística, ritualizada y expositiva. En este sentido, los autores argumentan que estas innovaciones promueven los vínculos con el conocimiento y con el aprendizaje apoyado en la 
comprensión, la creatividad y la metacognición, generando climas de aprendizaje basados en el diálogo y la participación. Por último, los autores expresan que las innovaciones se construyen en determinada institución e involucran a varios actores; se basan en evidencias científicas, saberes prácticos y experiencias de docentes e investigadores; estas son reales, se han implementado y es posible encontrar ejemplos concretos de cómo funcionan, e igualmente son viables, prácticas y adaptables a otros contextos.

En concordancia con lo anterior, Libedinsky (2016), considera que las innovaciones didácticas emergentes son propuestas que generan los docentes, que están caracterizadas por la oposición y ruptura con prácticas vigentes consolidadas y profundamente ensambladas con el contenido curricular disciplinar. Las mismas se implementan en un contexto y tiempo determinado, recuperando tradiciones y antecedentes, y atendiendo a los intereses culturales de los docentes y los estudiantes.

Entendida de este modo, la innovación es un proceso amplio, complejo, cuyos alcances exceden el ámbito del aula. Entonces podemos decir que la innovación tiene un carácter de transformación educativa y social; constituye un cambio que incide en algún aspecto estructural de la educación para mejorar su calidad.

Como se observa, los especialistas nos ponen ante un fuerte desafío, puesto que cuando se habla de innovaciones se involucran posturas epistemológicas y teóricas necesarias de explicitar cuando se emprende un camino pedagógico-didáctico que signifique una ruptura orientada a cambios reales. Estos deben atender a los requerimientos de una práctica situada, contextualizada, y suponen aprendizajes que solo se logran justamente innovando, un saber que debe hacerse praxis, es decir, un saber-hacer.

Según Zabalza (2003), innovar es introducir cambios justificados, por lo que innovar en docencia es aplicar tres condiciones: apertura, actualización, mejora. Es decir, es indispensable disponer de capacidad de adaptación y flexibilidad, y generar mejoras en los procesos educativos. El autor también destaca dos condiciones indispensables para la innovación en la universidad: practicidad y viabilidad (deben implicar resultados concretos y ser posibles de llevar a cabo).

... estamos ante una innovación cuando se pretende llevar a cabo un proceso de cambio bien fundamentado. Cambios viables y prácticos que están pensados desde la perspectiva de la mejora y actualización de nuestras actividades y dispositivos formativos en el seno de cada titulación. Cambios que serán documentados y evaluados. Propuesta de cambio que se ha formalizado en un proyecto que constituye su guía de desarrollo y compromisos. (Zabalza, 2003 p. 122)

En suma, se entiende a las innovaciones educativas como cambios planificados orientados a la mejora de los procesos de enseñanza y aprendizaje. Cambios que suponen nuevas formas de pensar y hacer en los contextos educativos, y que intentan potenciar la enseñanza y el aprendizaje. Múltiples condiciones interactúan en el desarrollo de innovaciones; se intentará recuperar, desde las expresiones de los docentes y estudiantes, algunas de ellas. 


\section{Materiales y métodos}

Se desarrolló un estudio cualitativo fenomenológico orientando al análisis de significaciones construidas por los actores que participan en diferentes innovaciones educativas en la universidad. Según Creswell y Poth (2007), los estudios fenomenológicos intentan captar los objetos de estudio tal como son percibidos por los participantes.

El tipo de muestreo fue intencional por criterio, ya que se consideró a docentes de la carrera de Licenciatura en Educación especial y Licenciatura en Psicopedagogía de la Universidad Nacional de Río Cuarto, docentes de la carrera de Licenciatura en Educación especial de la Universidad del Atlántico, y estudiantes avanzados de ambas carreras y universidades. Participaron 18 docentes, 11 profesores de la Universidad del Atlántico y 7 de la Universidad Nacional de Río Cuarto. La muestra de estudiantes estuvo compuesta por 32 personas, 22 de la Licenciatura en Educación especial de la Universidad del Atlántico (Colombia), y 10 de la Licenciatura en Psicopedagogía de la Universidad Nacional de Río Cuarto (Argentina); participaron 31 mujeres y 1 varón, cuyas edades varían entre 17 y 44 años.

Como instrumentos de recolección de datos se utilizaron entrevistas a docentes y cuestionarios abiertos a estudiantes. Los interrogantes orientadores, tanto de las entrevistas como de los cuestionarios, tenían el propósito de conocer cuáles son las concepciones que ambos actores tienen sobre qué es innovar y qué es hacerlo en educación, qué tipo de innovaciones realizan los docentes y de qué manera lo hacen. Igualmente, interesaba identificar cómo los estudiantes perciben dichas innovaciones; qué aspectos positivos y negativos pueden mencionar a modo de conocer aspectos facilitadores u obstaculizadores frente a procesos de innovación educativa; qué cambios creen que podrían llevarse a cabo a fin de lograr innovar en el aula, entre otros interrogantes que surgieron en el proceso de recolección de datos, como por ejemplo qué elementos debía tener una clase innovadora, qué vínculo podría establecerse entre innovación y conceptos como la creatividad, las TIC, las emociones, entre otros.

Para el análisis de los datos se realizaron procesos de codificación abierta, axial y selectiva (Soneira, 2006). Se construyeron las categorías a partir de las codificaciones y las comparaciones emergentes de los datos. Con base en planteamientos teóricos y estudios actuales se interpretaron los datos y resultados obtenidos. Para la construcción de las categorías se triangularon análisis de datos de los cuestionarios y las entrevistas.

El proceso de investigación se desarrolló conforme a pautas éticas establecidas para investigaciones en Ciencias Sociales. Se solicitó el consentimiento informado a los participantes; los datos fueron tratados de manera confidencial, preservando el anonimato.

\section{Resultados}

Los docentes y los estudiantes mencionan diferentes aspectos a modo de dimensiones que obstaculizan o facilitan los procesos de innovación. 
Mientras que, en algunos casos, algunas dimensiones parecen potenciar la innovación, en otros la obstaculizan, por eso se decide presentarlas de manera articulada, mostrando la complejidad y las diferentes perspectivas de los actores.

\section{Formación docente}

Los docentes sostienen que, para poder innovar en las aulas, es indispensable la formación docente continua y la actualización permanente. Los docentes de la UA sostienen que esto es necesario, pero es tomado como una barrera u obstáculo, ya que la formación docente se considera como algo muy costoso y que demanda recursos económicos a veces no disponibles.

"Los costos, en alguna medida, los costos planteados en formación docente también. Un docente que no se actualiza a veces no es porque no quiere, sino porque no tiene los recursos. Aquí en Colombia la cualificación docente no te la da el Estado, es decir, yo por lo menos estoy haciendo el doctorado becada, y esa es una situación casi que extraordinaria. Pero mis otras compañeras tienen que invertir para poderse capacitar, entonces han tenido que invertir, y estudios por fuera o dentro de Colombia, son muy costosos y eso a veces es lo que nos limita." (Docente "4", UA)

En relación con la formación docente, profesores de la UNRC coinciden en que la investigación es una de las funciones docentes más importantes para innovar, y tomándolo como factor facilitador mencionaron los programas existentes como los Proyectos de Investigación y de Innovación para la Mejora de la Enseñanza de Grado (PIIMEG), dependiente de la Secretaría Académica de la UNRC.

\footnotetext{
"Bueno, yo creo que para generar esas rupturas, esos quiebres en las concepciones se necesita conocimiento o formación, entonces bueno, me parece que algo primordial en las innovaciones educativas es justamente la formación docente, bueno eso creo que es primordial, tener, buscar constantemente, estar trabajando sobre la formación docente; bueno y acá en la universidad nuestra están, como que promueve mucho las innovaciones educativas y en muchos cursos de posgrados que van en esta dirección de establecer quiebres o rupturas con prácticas tradicionales y se generan muchos cursos de posgrados para que los docentes podamos acceder a nueva formación, a nuevos conocimientos, me parece que eso es fundamental." (Docente “4”, UNRC)
}

Según los docentes, formarse permite tomar conciencia de la necesidad de cambiar y otorga herramientas metodológicas y teóricas para poder hacerlo. En consonancia con lo anterior, las autoras Martini, Montebelli y Mancini (2009), refieren a que la poca o limitada formación pedagógica se considera un punto problemático para los procesos de innovación. Las autoras destacan que los docentes que muestran interés por actualizarse en cuestiones pedagógicas -como en los contenidos de las asignaturas-, tienen la necesidad de cambiar, modificar o transformar sus prácticas de enseñanza. Se considera acá que la formación docente debe ser uno de los puntos más importantes a tener en cuenta a la hora de pensar y poner en acción un proceso de innovación; el éxito de los mismos va a depender en 
gran medida de ello. Estudios previos también indican la importancia de la formación continua de los docentes para el desarrollo de procesos de trasformación educativa. En el estudio de Medina y Navío-Gámez (2018), se concluyó que los docentes otorgan importancia a la actualización y perfeccionamiento constante como un factor clave para la innovación educativa. Los resultados de la investigación de Pizzolito y Macchiarola (2015), indican que los profesores valoran positivamente la formación docente recibida en cursos, seminarios, talleres, grupos de estudio y carreras de posgrado en el campo educativo. Consideran que estas instancias formativas les permitieron desarrollar nuevas perspectivas, interrogantes y formas de pensar los procesos educativos. También es interesante señalar que los procesos formativos se retroalimentan de las prácticas innovadoras; según Pizzolito y Macchiarola (2015), "la formación que los docentes adquieren a partir de su participación en las innovaciones les permite construir conocimientos pedagógicos que se articulan con los saberes científicos propios del área de conocimiento en la que se han formado profesionalmente" (p.130).

Las relaciones entre innovación y formación se reconstruyen permanentemente de manera compleja y dialéctica. La innovación depende de la formación y los docentes se forman a partir de procesos innovadores. Las innovaciones generan transformaciones en los contextos y en los sujetos que la impulsan y sostienen.

\section{Redes y trabajo colaborativo}

Algunos docentes consultados también destacaron la importancia del trabajo colaborativo y la construcción de redes como modo de retroalimentación y aprendizaje mutuo.

\footnotetext{
“(...) el obstáculo fundamental es el docente con prácticas rutinarias, sobre todo creo que el individualismo, en el caso nuestro en la universidad, también a veces la desvalorización de la docencia en relación a otras funciones como puede ser la investigación, docentes que privilegian más la investigación que la innovación, entonces... que por eso la innovación en nuestra universidad, los PIIMEG que son proyectos de innovación e investigación, buscaron justamente constituirse como una política contrahegemónica a esta otra política de privilegio a la investigación, ¿cómo? Articulando la innovación con la investigación. También uno puede hacer investigación sobre la propia práctica, y poner en valor ese otro tipo de investigación." (Docente “1”, UNRC)
}

Martini, Montebelli y Mancini (2009), consideran como elementos importantes a la hora de pensar en el trabajo en equipo, en grupo o colaborativo, acciones como la apertura al diálogo, la responsabilidad, el respeto por lo que opinen los otros, la creatividad y el interés por el crecimiento tanto personal como grupal; desde la perspectiva profesional, estas son características que ayudan a los procesos innovadores. Las buenas relaciones, el trabajo en equipo, el conocerse previamente con los miembros del grupo y la heterogeneidad que cada uno pueda aportar son características de un ambiente de trabajo y un contexto ameno y favorable 
para abordar las problemáticas que surjan y poder acordar la mejor manera de resolverlas.

Pizzolito y Macchiarola (2015), también señalan que la conformación de equipos de trabajo colaborativo, interdisciplinarios e interinstitucionales son necesarios para intercambiar y construir conocimientos, para abrir espacios de discusión, para la articulación de saberes, para la interacción entre pares, para replantear dudas y cuestionar normas, supuestos o valores, que hasta el momento se creían consolidados y establecidos. El desarrollo de redes colaborativas de trabajo permite que se desarrollen trabajos complementarios en donde se puedan gestionar y compartir diversos conocimientos que estarán orientados a estrategias y objetivos comunes para la consolidación de aprendizajes grupales. Es por esto que las autoras consideran que las innovaciones educativas son complejas y generan la necesidad de realizar articulaciones interdisciplinarias y construir redes, ya que, de esta manera, desde la complementación y cooperación de las particulares miradas de cada uno de los actores involucrados se puedan abordar los problemas desde la multidimensionalidad que los caracteriza.

Las innovaciones educativas en la universidad parecen depender de la construcción de espacios de trabajo colaborativo entre docentes de las mismas cátedras y de otros espacios curriculares. El aislamiento y los problemas relacionales entre los grupos es una condición que obstaculiza las posibilidades de cambio, tal como consideran los participantes y se ha observado en otros estudios (De la Barrera, 2007).

\section{Dimensión institucional}

Según los testimonios de los docentes, se pudo visualizar que en la UNRC las políticas institucionales son vistas como favorecedoras de la innovación, mientras que algunos docentes de la UA consideran que las políticas institucionales son parte de los aspectos obstaculizadores en tanto generan procesos de burocratización.

"Yo creo que más que nada como factores que ayudan a la innovación me parece que mucho tiene que ver el contexto institucional; por ejemplo, acá las convocatorias de proyectos PIIMEG, que son los de la innovación en la enseñanza de grado, eso sin duda es un factor favorecedor porque a vos te da un marco en el cual vos podés incluir un proyecto específico tuyo de tu materia. Otra cosa favorecedora son las prácticas en colaboración o con otros, articuladas con otras materias, con otros docentes que por ahí compartís alumnos, entonces una innovación planteada de forma conjunta también tiene quizás como mejores resultados. El trabajo en equipo en la universidad, que es lo que por ahí cuesta tanto en otros niveles, realmente favorece la posibilidad de innovar. El tener vínculo con otras realidades, con otros contextos..." (Docente “3”, UNRC)

Los docentes de la UNRC consideran necesarias, favorables y muy importantes dichas políticas de innovación, porque le ofrecen a la universidad un amplio panorama de investigación y mejoramiento, a su vez que acreditan educación de calidad para la institución. Desde la Universidad del Atlántico, los docentes hacen hincapié en que 
las gestiones administrativas, cuestiones de currículum expedido por políticas educativas nacionales o regionales y la burocratización de la educación son factores que dificultan un proceso innovador.

"Se encuentran también con disposiciones en las gestiones administrativas que establecen un currículum cerrado, poco flexible, donde el maestro se encuentra atado de pies y manos, donde le cierran las posibilidades de que el maestro pueda cambiar, innovar, porque es mucho más fácil para la gestión educativa modelar, establecer esquemas, que dejar abierta la flexibilidad al docente, para controlar, para evaluar, para dar seguimiento. Es mucho más fácil que todo esté estandarizado, que todo esté modelado, que todo esté estructurado, es mucho más fácil... pero eso va en contra de las características de creatividad que le queremos pedir al maestro, resolver esa contradicción creo que también es un segundo elemento a tener en cuenta para facilitar la innovación.” (Docente “B”, UA)

Medina y Navío-Gámez (2018), subrayan el papel activo de las instituciones en la promoción del trabajo colaborativo y crítico orientado a la innovación. Proponen que las instituciones abran espacios de participación y generen sentido de pertenencia entre sus miembros. Según Pizzolito y Macchiarola (2015), las innovaciones generadas y promovidas por políticas institucionales ayudan a sostener los procesos innovadores a partir de experiencias de aprendizaje colaborativo entre docentes, de instancias de construcción de conocimientos implícitos y explícitos, y de evaluación de propuestas.

\section{Múltiples tareas, masividad y falta de tiempo}

La falta de tiempo y el desarrollo de múltiples tareas de manera simultánea es para los docentes un factor obstaculizador de la innovación. Igualmente, el dictado de clase con grupos numerosos de estudiantes también aparece como un aspecto que dificulta el desarrollo de innovaciones educativas.

"El tiempo; a veces uno tiene que cumplir tiempos académicos, tiempo para entregar notas, tiempos para desarrollar un contenido, una unidad, la dinámica de la universidad, nosotros, bueno yo no sé si usted se ha dado cuenta, yo creo que de los semestres este es el que menos hemos visto cosas, pero aquí somos una universidad que tiene unos instantes y unos momentos diferentes que de una $\mathrm{u}$ otra forma afectan los tiempos, los espacios e inciden en que esos procesos de innovación no se lleven a cabo." (Docente “G”, UA)

Resultados similares se han observado en otros estudios, muchos docentes consideran que la superposición de actividades y el escaso tiempo para la planificación y la reflexión dificultan la innovación. Medina y Navío-Gámez (2018), consideran que el sistema educativo tiene ciertas características que limitan la innovación: exceso de horas dentro del aula y numeroso trabajo administrativo. Esta sobrecarga afecta sobre la planificación de innovaciones y la institucionalización de cambios educativos. Martini, Montebelli y Mancini (2009), también han señalado que clases con grupos numerosos de estudiantes pueden dificultar el desarrollo de innovaciones, la participación, la interacción entre grupos y la evaluación de los cambios. 
La disponibilidad de tiempo genuino para la planificación y la evaluación permanente de las innovaciones dificulta el sostenimiento de los cambios y las mejoras en los contextos educativos. Las innovaciones en la enseñanza demandan muchos esfuerzos de los docentes, que algunas veces no son reconocidos en los ámbitos universitarios.

\section{Resistencia al cambio}

Tanto los docentes como los estudiantes manifiestan que la resistencia al cambio y el temor de lo nuevo afectan negativamente las innovaciones.

“El primer obstáculo que tú te encuentras, aunque haya un poco de predisposición
y temor al cambio, es la cantidad de documentación que les están exigiendo; no les
permite innovar porque se centran en las minucias de la escritura y no significa que
sistematizar esté mal, quiero decir que se le resta importancia al trabajo real con
el niño dentro del aula para llenar una cantidad de documentos, entonces cuando
hablo de eso, hablo de burocratización de la enseñanza, y hablar de burocratización
de la enseñanza es hablar de un sistema de educación cerrado que no es flexible
respecto a la educación, entonces hay que pensar en innovar pero no solamente
desde el aula, sino también desde los entes que regulan esa educación." (Estudiante
UA)

Las expresiones de los participantes se vinculan con las consideraciones que Martini, Mancini y Montebelli (2009), quienes sostienen respecto de actitudes negativas hacia el cambio, y las resistencias de docentes y estudiantes como obstáculos para la innovación: "El hecho de dedicarse a las tareas habituales implica mayor seguridad en el desarrollo de los aprendizajes, como contrapartida de toda acción alterada o modificada que un proyecto innovador representa" (p. 90).

Frente al temor al cambio y las resistencias de los actores, Medina y Navío-Gámez (2018), proponen estrategias institucionales que generen un mandato explícito de innovar, empoderen a los docentes y permitan que rompan con la enseñanza tradicional y los estimule a generar cambios educativos. Pizzolito y Macchiarola (2015), también destacan la importancia de las políticas institucionales orientadas al desarrollo de innovaciones educativas. Según las autoras, las políticas académicas dirigidas a impulsar, apoyar, financiar, comunicar y sostener en el tiempo las innovaciones en la enseñanza constituyen una condición de existencia de los procesos innovadores y explican el sentido de los mismos.

\section{Diagnóstico, participación y relación pedagógica}

Pizzolito y Macchiarola (2015), sostienen que todo movimiento innovador se inicia cuando los docentes reflexionan acerca de los procesos de enseñanza y se preguntan respecto de su accionar, advirtiendo problemas, posibilidades de cambio y desnaturalización de lo cotidiano.

En este sentido, los docentes de la Universidad del Atlántico han subrayado la necesidad de procesos de diagnóstico en las clases para la planificación de innovaciones. 
"Yo pienso que lo primero que hay que hacer es un diagnóstico de la caracterización de los estudiantes, igualmente el contexto sociocultural en el cual estamos inmersos, partiendo de eso, pienso que innovar es no seguir las pautas tradicionales de la enseñanza, es proponer nuevas alternativas acordes con los estudiantes con sus necesidades y con sus intereses... (Docente "E”, UA)

La innovación, en tanto proceso de planificación y con claros objetivos educativos, necesariamente debe partir de una adecuada valoración de las potencialidades y dificultades del grupo clase. Los docentes y los estudiantes de la Universidad del Atlántico también destacan la importancia de la participación y la comunicación entre docentes y estudiantes para el desarrollo de innovaciones educativas.

“(...) tenemos que tener en cuenta que para ser innovadores en educación hay que tener una mayor participación del estudiante, ese estudiante activo que crea, que transforma y que el docente sea orientador, no dictador, sino orientador, que es el que está ahí como que pendiente del proceso." (Docente "I”, UA)

"Interactuar mucho, despertar su interés por medio de actividades donde el estudiante utilice su creatividad y proponer por medio de la didáctica clases extraordinarias." (Estudiante UA)

Podemos inferir que lo que consideran los estudiantes como condición favorecedora para innovar está relacionado con el tipo de clase o de participación que se le propone desde una cátedra o un determinado profesor. Esto hace relacionarlo con las categorías que emergieron desde la investigación sobre factores favorecedores, que proponen las autoras Martini, Montebelli y Mancini (2009). Las autoras investigaron y a una de las categorías le proporcionaron el nombre de "actitudes positivas de los alumnos"; esta aporta que tales actitudes que tienen los estudiantes al respecto de la participación en distintos espacios y la respuesta positiva que tienen frente al uso de nuevos elementos y estrategias para su aprendizaje son elementos favorecedores para una innovación educativa, ya que el compromiso y el entusiasmo permiten un buen desarrollo de las propuestas de los equipos de trabajo docentes.

En este sentido, la falta de diálogo y participación en los contextos áulicos y el desconocimiento de los procesos de aprendizaje que desarrollan los estudiantes (estilos, estrategias, modos de aprender), son factores que obstaculizan los procesos innovadores, tal como lo consideran estudiantes y docentes.

"Continuar con un modelo tradicional a la hora de dar las clases como, por ejemplo, continuar con la misma metodología de enseñanza, centrándose mucho en el contenido sin saber si realmente el alumno está comprendiendo lo que se está dando; muchas veces se enseña, pero no se aprende." (Estudiante UNRC)

"Pretender que todos los estudiantes aprendan de la misma forma con las mismas estrategias." (Estudiante UA)

"No cubrir todos los ritmos de aprendizaje." (Estudiante UA)

En suma, el diagnóstico inicial de las situaciones educativas es fundamental para la generación de cambios. El reconocimiento de problemas y la búsqueda de alternativas son indispensables para la innovación educativa. Del mismo modo, docentes y estudiantes reconocen la importancia de los intercambios, la participación y el dialogo 
como factores que contribuyen a la innovación. El reconocimiento de particularidades en los procesos de construcción de conocimientos (estilos, estrategias y ritmos), también es indispensable, tal como subrayan los estudiantes.

\section{Más allá de las aulas}

La mayoría de las prácticas innovadoras identificadas por los estudiantes refieren a proyectos fuera de los contextos áulicos. Los participantes se refieren a propuestas educativas vinculadas a prácticas sociocomunitarias, proyectos de investigación, trabajos de campo y pasantías. Estas propuestas, si bien tienen articulación con los contenidos curriculares de las asignaturas, se refieren a acciones concretas en contextos fuera de las aulas.

\footnotetext{
"Por ejemplo, en un momento hacer práctica socio comunitaria en una materia era una innovación realmente, esto de poder vincular al alumno con el medio, con la necesidad, responder y armar grupos de trabajo y demás, funcionó como una innovación." (Docente “3”, UNRC)

"La participación en proyectos educativos. En micropasantías, en talleres, seminarios." (Estudiante UNRC)

"Las prácticas, trabajos en campo, videos, conferencias, talleres, etc." (Estudiante UNRC)

"Trabajos en equipo donde te permitan investigar, ir a lugares para conseguir esa información." (Estudiante UNRC)
}

En otros estudios (Elisondo, Donolo y Rinaudo, 2009; Elisondo y Donolo, 2015; Elisondo y Melgar, 2017), también se encuentra que las propuestas más allá de las aulas se configuran como espacios para la creatividad y la innovación, en tanto suponen procesos de resolución de problemas complejos y búsqueda de alternativas. Las propuestas más allá de las aulas permiten construir conocimientos a partir de la articulación entre teorías y prácticas concretas. Los estudiantes entrevistados acuerdan en que las actividades extra-académicas contribuyen -en gran medidaa su formación, ya que les permiten acceder a conocimientos no solo teóricos sino también procedimentales y actitudinales. Aquellos que participan en proyectos de extensión destacan la importancia de las acciones que realizan en tanto oportunidades de adquirir experiencias diversas y conocimientos teóricos y prácticos. Sin embargo, consideran que las motivaciones que los guían a realizar estas actividades van mucho más allá de intereses personales. Realizar contribuciones sociales y comunitarias es la finalidad principal que persiguen estos estudiantes. Las actividades en contextos reales generan interacciones con otras personas y objetos de conocimiento, permiten cuestionar conocimientos académicos y generar aprendizajes complejos en interacción con problemas genuinos. En este sentido, se subraya el valor de las prácticas socio-comunitarias como espacios genuinos de aprendizaje en territorios sociales diversos (Macchiarola y Juárez, 2014; Tarifa, 2013). 


\section{Innovación y tecnologías}

Respecto de las relaciones entre innovación y TIC aparecen diferentes posturas entre los participantes. Mientras que para algunos son elementos indispensables para generar procesos innovadores en la universidad, otros ponen en duda su efectividad si el uso de tecnologías no está acompañado por objetivos orientados al cambio. Es decir, la mera incorporación de tecnologías no garantiza innovaciones, los cambios dependen de las perspectivas de los actores y los objetivos educativos que se plantean.

\footnotetext{
"Mira que también es importante que no todo tiene que ser tecnología, (...) cuando aprenden las tecnologías aplicadas a la discapacidad, ¿cierto?, tienen que saber que existen las tecnologías de apoyo para los diferentes requerimientos y necesidades que tienen las personas para tener su autonomía, su independencia, para acceder a información, para comunicarse, pero yo no se lo puedo dar práctico porque ni siquiera la misma institución tiene un laboratorio, debiera tener laboratorio de tecnologías más completo y no simplemente el acceso al computador." (Docente "A", UA)

"Bueno, ahí no hay una relación necesaria, es decir, uno puede ser enseñando de la misma manera, lo mismo y de la misma forma donde la tecnología simplemente adorna y demuestra más amena la práctica. O bien, sí creo, que las tecnologías son un potencial interesante para hacer innovaciones, bien usadas, usando todo el potencial que tienen las tecnologías, pueden ser una plataforma para innovar (...) te rompe la linealidad del aprendizaje, la posibilidad de que el alumno aprenda más allá del aula, más allá de los tiempos escolares, de los tiempos académicos, eso implica rupturas en los espacios y tiempos de los aprendizajes." (Docente "1", UNRC)
}

La mayoría de los estudiantes de la UA percibe a las tecnologías como herramientas propicias para la innovación y reconocer su valor en la sociedad actual. Los estudiantes, particularmente los de la UA, consideraron a las TIC como factor favorecedor de la innovación, pues argumentan que por medio de ellas están más conectados con sus docentes y les permiten asumir un papel más protagónico en sus aprendizajes.

Sin embargo, los participantes también perciben obstáculos vinculados al acceso a las tecnologías y también a los objetivos que subyacen a la incorporación de estos recursos. Es decir, si las prácticas educativas siguen sustentándose en perspectivas que asignan un papel pasivo a los estudiantes, y al docente el rol de transmisor de las tecnologías, solo apoyan los procesos de reproducción sin generar espacios para la innovación. Sierra-Llorente, Palmezano-Córdoba y Romero-Mora (2018), señalan dificultades vinculadas a la incorporación de TIC en los procesos educativos: falta de infraestructura y recursos económicos para la instalación y el mantenimiento de equipos, necesidad de formación docente continua y capacidad de manejo de recursos y programas actuales.

"El uso constante del PowerPoint para dar las clases, me parece que no ayuda, es una lectura directa del PowerPoint y no agrega nueva información." (Estudiante UNRC)

"Falta de recursos y acceso a tecnologías es un obstáculo para la innovación.” (Estudiante UA) 
Existen diversas posturas sobre el uso de las TIC. Las TIC han posibilitado otras maneras de aprender, otros medios de transmitir la información, la difusión masiva de esta, nuevos espacios de enseñanza, otro tipo de interacción entre docente-estudiante, nuevas estrategias, estilos y técnicas de aprendizaje. Las tecnologías amplían las posibilidades de interacciones entre personas y de acceso a conocimientos diversos (Dussel y Reyes, 2018). Igualmente, permiten comunicaciones en diferentes tiempos y espacios, facilitando procesos innovadores al generar rupturas en las formas de aprender y enseñar. Este estudio coincide con los participantes en que las tecnologías pueden contribuir al desarrollo de innovaciones solo si se apoyan en creencias y objetivos orientados a la trasformación en las formas de enseñar y aprender. Además, se considera que es preciso que las instituciones educativas generen proyectos de transalfabetización (Ipri, 2010), que integren a las tecnologías en los complejos procesos de enseñanza y aprendizaje en la Universidad (Elisondo y Donolo, 2014).

\section{Discusión}

Las expresiones de los participantes dan cuenta de la complejidad de los procesos innovadores y de los múltiples condicionantes que interactúan en los cambios educativos. La mayoría de los testimonios vinculan a las innovaciones educativas con la idea de cambiar, mejorar, transformar, romper con estructuras tradicionales. Tanto docentes como estudiantes consideran de suma importancia la implementación de innovaciones en las clases universitarias, permitiendo romper con prácticas tradicionales que provocan monotonía en la práctica docente y desmotivación en los estudiantes.

Los docentes mayormente destacaron como aspectos favorecedores a la formación docente, la investigación de las innovaciones para producir más innovaciones, el trabajo en equipo y políticas institucionales. En cuanto a los aspectos obstaculizadores, también encontramos que las políticas institucionales, en términos de burocratización son problemáticas. También remarcan el hecho de la falta de tiempo, que está relacionada con la multiplicidad de tareas, la resistencia al cambio de sus estudiantes, el costo de la formación docente -en el caso de los profesores colombianosy la masividad de estudiantes por asignaturas.

Con relación a las experiencias que tienen sobre el rol de sus docentes en el aula, los estudiantes consideran que aún algunos docentes siguen regidos por un modelo tradicional de educación; reconocen también que otros mantienen un discurso constructivista, pero siguen actuando de manera tradicional. Por otro lado, reconocen que hay docentes que toman una posición de guía en el proceso de enseñanza-aprendizaje, brindándoles a los estudiantes herramientas de las que disponen para optimizar este proceso. Los estudiantes también consideran como aspectos positivos para que se desarrollen los procesos de innovación educativa la incorporación de las TIC, las prácticas en espacios extracurriculares como pasantías, seminarios, talleres, entre otros, y 
también consideran importante el intercambio de información con otros profesionales u otras áreas de conocimiento.

Prácticas socio-comunitarias, semilleros de investigación, pasantías, proyectos de extensión, parecen generar contextos de enseñanza y aprendizaje innovadores que rompen con las prácticas tradicionales y habilitan procesos complejos de construcción de conocimientos articulados con la realidad. Podemos ver que estos aspectos forman parte también de las propuestas innovadoras que ellos realizan para mejorar su proceso de aprendizaje. En cuanto a los aspectos negativos, los estudiantes remarcan la postura tradicional de sus docentes a la hora de enseñar, la burocratización de la información, el temor y la resistencia al cambio, y el mal uso de las TIC como principales obstáculos.

Como futuras líneas de investigación se podría ampliar la temática de la innovación educativa mediante el estudio de las prácticas profesionales en diferentes carreras de grado. También es interesante investigar percepciones de docentes y estudiantes a la evaluación en contextos innovadores. En futuros estudios es necesario profundizar los análisis respecto del papel de las TIC en las innovaciones que se desarrollan en las universidades. De la misma manera, en nuevas investigaciones es necesario construir procesos de triangulación metodológica que permitan arribar a interpretaciones más sólidas respecto de los procesos innovadores en la universidad.

Los conocimientos construidos en la presente investigación, a pesar de ser limitados y acotados a una muestra particular, ofrecen interesantes líneas de investigación e intervención para el diseño de innovaciones en las universidades. Reconocer las condiciones que facilitan y obstaculizan los cambios es relevante en la construcción de nuevas propuestas transformadoras. Reflexionar sobre las innovaciones y generar espacios alternativos para la enseñanza y el aprendizaje en la universidad es un desafío permanente para docentes, investigadores y gestores de políticas educativas.

\section{REFERENCIAS}

Creeswell, J., \& Poth, C. (2007). Qualitative Inquiry and Research Design: Choosing among Five Approaches. Londres: Sage publications.

Delgado-Coronado, S. (2019). Perspectivas en torno a la formación docente y la posibilidad de una capacitación y actualización constante: una mirada desde los actores de una universidad mexicana. Revista Panorama, 13(24), 34-41. Recuperado de: https://journal.poligran.edu.co/index.php/panor ama/article/view/1204

De la Barrera, S. (2007). Colaboración entre profesores ¿Quién dice que es fácil? Colección de Cuadernillos de actualización para pensar la Enseñanza Universitaria. Río Cuarto: Universidad Nacional de Río Cuarto. Recuperado de: https://www.unrc.edu.ar/unrc/academica/docs /publicaciones/delabarrera-may07.pdf

Dussel, I. \& Reyes, B. (2018) ¿Nuevas formas de enseñar y aprender? Las posibilidades en conflicto de las tecnologías digitales en la escuela. Perfiles 
Educativos, 40(Especial), 142-178. Recuperado de: http://perfileseducati vos.unam.mx/iisue_pe/index.php/perfiles/article/view/59182

Elisondo, R., Donolo, D., \& Rinaudo, M. (2009). Contextos de educación en la universidad: perspectivas de los alumnos potencialmente más creativos. Revista Iberoamericana De Educación, 49(6), 1-11. Recuperado de: https: //rieoei.org/RIE/article/view/2054

Elisondo, R. \& Donolo, D. (2014). Creatividad y alfabetización informacional. El desafío en cuatro propuestas. Panorama, 8(15), 23-33. Recuperado de: https://journal.poligran.edu.co/index.php/panorama/article/view/547

Elisondo, R. \& Donolo, D. (2015). Creatividad en la Universidad. Cuadernos de Educación. Tenerife: Sociedad Latina de Comunicación Social. Recuperado de: https://issuu.com/revistalatinadecomunicacion/docs/cd e04

Elisondo, R. \& Melgar, F. (2017). Creatividad y Universidad. Estudios más allá de los muros. Málaga: Eumed. Recuperado de: http://www.eumed.net/lib ros/img/portadas/1668.pdf

Ipri, T. (2010). Introducing transliteracy. What does it mean to academic libraries? College \& Research Libraries News, 71(10), 532-567.

Libedinsky, M. (2016). La innovación educativa en la era digital. Paidós: Buenos Aires.

Macchiarola, V. \& Juárez, M. (2014) Experiencias de prácticas socio-comunitarias en la universidad. Recorridos, emergencias y desafios de una innovación pedagógico-social. Río Cuarto: UNIRIO Editora.

Martini, C., Montebeli, E., \& Mancini, A. (2009) Las condiciones facilitadoras y obstaculizadoras de innovación en la universidad. En Macchiarola, V.: Rupturas en el hacer y el pensar. Río Cuarto: UNIRIO Editora.

Medina, J., \& Navío-Gámez, A. (2018). Concepciones sobre innovación educativa. ¿Qué significa para los docentes en Chile? Profesorado, Revista de Currículum y Formación del Profesorado, 22(4), 71-90. Recuperado de: https://revistaseug.ugr.es/index.php/profesorado/article/view/8395

Norman-Acevedo, E. (2019). Nuevos lenguajes para aprendizaje virtual, herramientas para los escenarios de aprendizaje. Revista Panorama, 13(24), 5-7.

Pizzolito, A., \& Macchiarola, V. (2015) Un estudio sobre cambios planificados en la enseñanza universitaria: origen y desarrollo de las innovaciones educativas. Innovación Educativa, 15 (67), 111-134. Recuperado de: http://www.scielo.org.mx/scielo.php?script=sci_arttext\&pid=S1665 $-26732015000100007$

Rivas, A., André, F., Delgado, L., Aguerrondo, I., Anijovich, R., Furman, M., ... \& Vota, A. (2017). 50 innovaciones educativas para escuelas. Lima: Centro de Implementación de Políticas Públicas para la Equidad y el Crecimiento. Recuperado de: https://www.cippec.org/wp-content/uploads/2017/08/ 978-950-46-5369-1-CIPPEC-50-Innovaciones-educativas.pdf

Sierra-Llorente, J., Palmezano-Córdoba, Y., \& Romero-Mora, B. (2018). Causas que determinan las dificultades de la incorporación de las TIC en las aulas de la clase. Revista Panorama, 12(22), 32-41. Recuperado de: https://jour nal.poligran.edu.co/index.php/panorama/article/download/1064/1037

Soneira, A. (2006). La teoría fundamentada en los datos (Grounded Theory) de Glaser y Strauss. En Vasilachis, I.: Estrategias de investigación cualitativa (pp.153-173). Buenos Aires. 
Tarifa, R. (2013). Incorporación de las prácticas socio-comunitarias al currículo universitario. Revista Contextos de Educación, 13(15), 39-46.

UNESCO Perú. (2016). Innovación educativa. Herramientas de apoyo para el trabajo docente. Lima: UNESCO. Recuperado de: http://repositorio.minedu.gob.pe/bitstream/handle/MINEDU/513 5/Innovaci\%C3\%B3n\%20educativa.pdf?sequence $=1$

Zabalza, M. (2003) Innovación en la enseñanza universitaria. Contextos Educativos. Revista de Educación, 6, 113-136. 